\title{
Human and physical factors involved in indigenous processing for value addition of rice
}

\author{
BIJOYLAXMI BHUYAN AND MIRA KALITA
}

Received: 23.07.2014; Accepted: 22.11.2014

See end of the paper for authors' affiliations

\section{MIRA KALITA}

Department of Family Resource Management, College of Home Science, Assam Agricultural University, JORHAT (ASSAM) INDIA
ABSTRACT : In Assamese culture, many bi-products of rice are prepared with indigenous techniques and are under women's work domain. Preparations of these products are highly skillful and are labour intensive. These ready to eat nutritious products from modern 'health food' viewpoint are highly acceptable but quality is observed to be not always satisfactory. This could be due to paying of less attention to economic use of human factors and comfort in the work place. These aspects of work provide comfortable work condition and consequently promote health and efficiency of the worker that help to maintain quantity and quality of products. In this context, the study was undertaken to analyse human factors and physical factors in work place involved in processing of aforesaid products. Study revealed that 86 per cent of the workers complained of high to medium discomfort in the lumbo sacral region, which might be due to adoption of awkward postures. Rating perceived exertion of the workers was reported as 'high' indicating the work to be fatiguing, the reason might be low level of illumination in the kitchen, time consuming aspects/factors at work and need to attend to other household tasks simultaneously. In all, the physical conditions in the work place were found to be less conducive to workers. To improve human and physical factors for economic upliftment of rural women involved in self-help groups in Assam in particular, proper interventions could be planned.

KEY WORDS: Rice, Indigenous processing, Value addition of rice

- HOW TO CITE THIS PAPER : Bhuyan, Bijoylaxmi and Kalita, Mira (2014). Human and physical factors involved in indigenous processing for value addition of rice. Asian J. Home Sci., 9 (2) : 633-635. 